\title{
A Nonlinear Mechanical Model for Predicting the Dynamics Response of Materials under a Constant Loading
}

\author{
Marc Delphin Monsia \\ Département de Physique, Université d'Abomey-Calavi \\ Abomey-Calavi, 09 B.P. 305, Cotonou, Bénin \\ Tel: 229-955-681-87 E-mail: monsiadelphin@yahoo.fr
}

Received: December 5, 2011 Accepted: December 15, 2011 Published: January 1, 2012

doi:10.5539/jmsr.v1n1p90

URL: http://dx.doi.org/10.5539/jmsr.v1n1p90

\begin{abstract}
The paper is designed to provide a theoretical mechanical model taking into account elastic, viscous and inertial nonlinearities simultaneously, for predicting the time dependent response of some materials under a constant loading. Numerical illustrations are carried out to investigate the effects of rheological coefficients action on the model.
\end{abstract}

Keywords: Lambert-type equation, Mathematical model, Nonlinear stress-strain relationship, Viscoelasticity, Voigt model

\section{Introduction}

In mechanics mathematical models are important analytical tools for predicting and simulating the material response under various solicitations and for optimizing the design. Most of materials undergo time dependent deformations under applied external forces. These materials are characterized by both combined elastic and viscous behaviors, so that they cannot be described by the simple theory of elasticity. In this regard the use of the theory of viscoelasticity is then suggested. In viscoelasticity, linear rheological models are often used to quantify materials properties under small deformations assumption. The linear theory of viscoelasticity becomes for large deformations, however, inapplicable. Thus, nonlinear rheological models are required for characterizing material functions under large deformations. The linear theory of viscoelasticity is usually expressed in the Boltzmann single integral or in the differential form. But, a standard framework does not exist in nonlinear viscoelasticity. To construct nonlinear viscoelastic models, it is admissible to modify and extend the linear theory to higher order stress or strain terms. In this perspective, a number of viscoelastic models have been performed for quantifying the nonlinear material properties. Since a complete characterization of viscoelastic materials is very difficult, due to the necessity to take into account elastic, viscous and inertial nonlinearities simultaneously (Bauer et al. 1979; Bauer 1984), only the elastic or viscous nonlinearity is often taken into consideration in most existing analytic rheological models (Monsia 2011a,b,c), so that the inertial contribution is neglected. Moreover, a few mathematical models formulated with constant-value rheological material parameters exist. By using a second-order elastic spring in series with a classical Voigt element, that is a nonlinear standard solid model, Monsia (2011a) developed a hyperlogistic-type equation for representing the nonlinear stress versus time relationship of a variety of materials. Recently, Monsia (2011b) derived from a nonlinear standard solid model consisting of a polynomial elastic spring in series with a classical Voigt element, a single nonlinear differential constitutive equation for the prediction of time dependent stress response of some viscoelastic materials. Recently again, Monsia (2011c) formulated a nonlinear four-parameter rheological model consisting of a nonlinear Voigt model in series with a classical linear Voigt element with constant material coefficients to describe the nonlinear stiffening response of the initial low-load region and the softening behavior of a variety of viscoelastic materials. More recently, Monsia (2011d) developed a nonlinear generalized Maxwell model consisting of a nonlinear spring connected in series with a nonlinear dashpot obeying a power law with constant material parameters for representing the time dependent properties of some materials. Therefore, according to Bauer (1984) these models cannot give a complete description of viscoelastic materials, in the understanding that these materials are characterized by strong elastic, viscous and inertial nonlinearities. Advanced mechanical models are then required for a complete and satisfactory characterization of viscoelastic materials properties. 
Their constitutive equations must in that case relate stress, strain and their higher time derivatives (Bauer, 1984). In this perspective, to overcome the mathematical complexities in modeling of viscoelastic materials, Bauer (1984) developed a theory based on the classical linear Voigt model for a complete description of rheological properties of arterial walls. The Bauer's theory (1984) is intended to give satisfactorily and simultaneously account of high elastic, viscous and inertial nonlinearities characterizing a viscoelastic material. The approach consists essentially to decompose the total stress due to the external exciting force as the sum of three components, that is to say, the elastic, viscous and inertial stresses and to express the pure elastic stress as a nonlinear function of deformation. The pure viscous and inertial stresses are then formulated as a first and second time derivatives of a similar function of deformation to the nonlinear elastic function, respectively. A major theoretical difficulty in the use of the Bauer's theory (1984) consists of the determination of appropriate nonlinear elastic restoring force function that tends towards the expected linear elastic behavior for small deformations. In the Bauer's study (1984), the pure elastic stress is expanded in a power series of strain, the pure viscous stress is developed as a first time derivative of a similar power series of strain, and the pure inertial stress is expressed as a second time derivative of a similar power series of strain. Many authors (Armentano et al. 1995; Gamero et al. 2001), after, have used the Bauer's stress decomposition method (1984), consisting to express the stress as a sum of three elementary stresses, for a complete characterization of arterial behavior. Recently, Monsia (2011e), using the Bauer's method (1984), developed a hyperlogistic equation that is useful for representing the time dependent behavior of some viscoelastic materials. In Monsia (2011e), following the Bauer's theory (1984), the pure elastic stress is developed in an asymptotic expansions in powers of deformation, the pure viscous stress is expressed as a first time derivative of a similar asymptotic expansions in powers of deformation, and the inertial stress is formulated as a second time derivative of a similar asymptotic expansions in powers of deformation. The present paper is designed to develop a consistent mathematical one-dimensional model with constant material coefficients applicable for characterizing the time dependent deformation behavior of a variety of materials under a constant loading. The model is derived from the Bauer's theory (1984) that allows taking into account elastic, viscous and inertial nonlinearities simultaneously. The resulting evolution equation is a Lambert-type nonlinear ordinary differential equation that can be solved in closed-form solution by using suitable boundary and initial conditions. Numerical examples are carried out for illustrating the sensitivity of the model to material parameters.

\section{Formulation of the Mathematical Model}

\subsection{Theoretical Considerations}

We develop in this part the evolution equation of the mechanical system under consideration. Most materials exhibit when they are subjected to applied forces large time dependent deformations and manifest then nonlinearity properties, so that the use of appropriate nonlinear viscoelastic theory is required. In this regard the Bauer's theory becomes an important mathematical tool for taking into consideration the elastic, viscous and inertial nonlinearities simultaneously. The Bauer's approach brings significant modifications and extensions to the classical mechanical Kelvin-Voigt model in sight of a complete and satisfactory characterization of viscoelastic materials. The first significant modification herein consists as indicated in Monsia (2011e), to introduce a nonlinear restoring spring force function $\varphi(\varepsilon)$ of deformation $\varepsilon$ for capturing the pure elastic component of the stress induced in the material studied. Then, the pure elastic constitutive equation may be written as

$$
\sigma_{e}=a \varphi(\varepsilon)
$$

where $a$ is a stiffness coefficient. The second important modification as mentioned in Monsia (2011e), involves the fact that the pure nonlinear viscous constitutive equation is then directly given by

$$
\sigma_{v}=\frac{d}{d t}[b \varphi(\varepsilon)]
$$

where $b$ is a viscosity module. The third significant modification consists then to derive from $\varphi(\varepsilon)$ the nonlinear inertial stress component in the form

$$
\sigma_{i}=\frac{d^{2}}{d t^{2}}[c \varphi(\varepsilon)]
$$


where $c$ is an inertia module. Note that in this present one-dimensional model, the stresses and strain are scalar functions and the coefficients $a, b$ and $c$ are time independent material parameters. Thus, noting $\sigma_{t}$ the total stress due to the external exciting force acting on the material, the superposition of elastic stress, viscous stress and inertial stress is given by (Monsia 2011f)

$$
\ddot{\varepsilon} \frac{d \varphi}{d \varepsilon}+\dot{\varepsilon}^{2} \frac{d^{2} \varphi}{d \varepsilon^{2}}+\frac{b}{c} \dot{\varepsilon} \frac{d \varphi}{d \varepsilon}+\frac{a}{c} \varphi(\varepsilon)=\frac{1}{c} \sigma_{t}
$$

where the dot over the symbol denotes a differentiation with respect to time and the inertial module $c$ is different from zero. Equation (4) assesses the differential constitutive relationship between the total external stress $\sigma_{t}$ and the resulting strain $\varepsilon(t)$ for a given nonlinear function $\varphi(\varepsilon)$. It is needed, at this stage of modeling, to identify the nonlinear function $\varphi(\varepsilon)$. In the present paper, the nonlinear spring force function $\varphi(\varepsilon)$ is chosen as in Monsia (2011f) in the following hyperbolic law

$$
\varphi(\varepsilon)=\frac{\varepsilon}{\varepsilon-1}
$$

Thus, using Equation (5), Equation (4) may be written as

$$
\ddot{\varepsilon}-\frac{2}{\varepsilon-1} \dot{\varepsilon}^{2}+\frac{b}{c} \dot{\varepsilon}-\frac{a}{c} \varepsilon(\varepsilon-1)=-\frac{1}{c}(\varepsilon-1)^{2} \sigma_{t}
$$

Equation (6) denotes a second-order nonlinear ordinary differential equation in $\varepsilon(t)$ for a given total stress $\sigma_{t}$. It represents in differential form the constitutive stress-strain relationship which obeys the material studied.

\subsection{Dimensionalization}

Noting that the strain $\varepsilon(t)$ is a dimensionless quantity, the above material parameters used in Equation (6) have then the following dimensions. If $M, L$ and $T$ denote the mass, length and time dimension respectively, the dimension of the stress becomes $M L^{-1} T^{2}$. Therefore, the dimension of $a$ is given by $M L^{-1} T^{2}$, that of $b$ varies as $M L^{-1} T^{-1}$, and that of $c$ varies as $M L^{-1}$ (mass per unit length).

\subsection{Solution using a constant loading control}

We derive in this section the closed form solution of Equation (6) allowing the description of the time dependent strain induced in the material studied. To that end, we consider that the material under consideration is subjected to a constant external exciting stress, to say $\sigma_{t}(t)=c s t e$. If we set

$$
\frac{\sigma_{t}}{c}=k
$$

where $k$ is a constant coefficient, Equation (6) becomes

$$
\ddot{\varepsilon}-\frac{2}{\varepsilon-1} \dot{\varepsilon}^{2}+\frac{b}{c} \dot{\varepsilon}-\frac{a}{c} \varepsilon(\varepsilon-1)=-k(\varepsilon-1)^{2}
$$

or

$$
\ddot{\varepsilon}-\frac{2}{\varepsilon-1} \dot{\varepsilon}^{2}+\lambda \dot{\varepsilon}-\omega_{o}^{2} \varepsilon(\varepsilon-1)=-k(\varepsilon-1)^{2}
$$

where $\lambda=\frac{b}{c}$, and $\omega_{o}^{2}=\frac{a}{c}$.

Equation (7) represents the time dynamics equation of the strain $\varepsilon(t)$ induced in the material under a constant external exciting stress. By introducing the auxiliary variable

$$
x=\varepsilon-1
$$


Equation (7) transforms, after a few algebraic manipulations, into

$$
\ddot{x}-2 \frac{\dot{x}^{2}}{x}+\lambda \dot{x}-\omega_{o}^{2} x-\left(\omega_{o}^{2}-k\right) x^{2}=0
$$

We can interpret each term present in Equation (9) following Monsia (2011f). The first term in Equation (9) is proportional to the basic inertial stress, the second to a nonlinear quadratic viscous stress, the third term to the linear viscous stress, the fourth term to the classical linear elastic stress and the last term to a quadratic nonlinear elastic stress. Equation (9) is a Lambert-type nonlinear ordinary differential equation that can be solved in closed-form solution by using an appropriate change of variable and suitable boundary and initial conditions that satisfy the time dynamics of the viscoelastic material considered.

2.3.1 Case A: $k=\omega_{o}^{2}$

In this particular case where $k=\omega_{o}^{2}$, Equation (9) becomes

$$
\ddot{x}-2 \frac{\dot{x}^{2}}{x}+\lambda \dot{x}-\omega_{o}^{2} x=0
$$

Note that in Monsia (2011e), a similar equation to (10) is already solved. Therefore, letting the following change of variable

$$
f=\frac{\dot{\varepsilon}}{\varepsilon}
$$

Equation (11) leads, after some algebraic manipulations, to the following Riccati nonlinear ordinary differential equation for the variable $f$ that possesses the strain rate dimension

$$
\dot{f}=f^{2}-\lambda f+\omega_{o}^{2}
$$

Using the following boundary conditions

$$
t \rightarrow 0, \lim f(t)=f_{o}
$$

and

$$
t \rightarrow+\infty, \lim f(t)=0
$$

the solution of Equation (10) may be written

$$
x(t)=x_{\max }[1+q \exp (-\lambda \delta t)]^{-1 / n}
$$

where

$$
\delta=\sqrt{1-4 \frac{\omega_{o}^{2}}{\lambda^{2}}}, f_{2}=\frac{\lambda}{2}(1-\delta), q=\frac{f_{o}}{f_{2}-f_{o}}, n=\frac{\lambda \delta}{f_{2}}
$$

and the condition

$$
t \rightarrow+\infty, \lim x(t)=x_{\max }=K
$$

By considering Equation (8) the desired time dependent strain can be written as

$$
\varepsilon(t)=1+K[1+q \exp (-\lambda \delta t)]^{-1 / n}
$$

Equation (14) predicts the strain versus time variation of the material under study as a hyperlogistic -type model that is well known to be powerful for describing mathematically any S curve shape. 


\subsubsection{Case B: $k \neq \omega_{o}^{2}$}

In this particular case where $k \neq \omega_{o}^{2}$, for solving Equation (9) the following substitution is needed

$$
x=y^{-1}
$$

in which $y \neq 0$. Equation (9) transforms, after a few algebraic manipulation as

$$
\ddot{y}+\lambda \dot{y}+\omega_{o}^{2} y=k-\omega_{o}^{2}
$$

Equation (16) denotes the well known second-order linear ordinary differential equation with a right-hand member different from zero. Integration yields for $y(t)$ the following solution

$$
y(t)=\frac{k}{\omega_{o}^{2}}-1+A_{1} \exp \left(r_{1} t\right)+A_{2} \exp \left(r_{2} t\right)
$$

where

$$
r_{1}=-\frac{\lambda}{2}(1-\delta)
$$

and

$$
r_{2}=-\frac{\lambda}{2}(1+\delta)
$$

are the two negative real roots of the characteristic equation

$$
r^{2}+\lambda r+\omega_{o}^{2}=0
$$

with

$$
\delta=\sqrt{1-4 \frac{\omega_{o}^{2}}{\lambda^{2}}}
$$

$A_{1}$ and $A_{2}$ are two integration constants determined by the initial conditions. By considering then the suitable initial conditions

$$
t=0, \varepsilon(t)=0
$$

and

$$
t=0, \dot{\varepsilon}(t)=f_{o}
$$

and using Equation (8) and Equation (15), the desired strain versus time relationship can be written as

$$
\varepsilon(t)=1+\frac{k}{\frac{\omega_{O}^{2}}{2}-1+\frac{1}{\lambda \sqrt{1-4 \frac{\omega_{o}^{2}}{\lambda^{2}}}}\left[\left(-\frac{k \lambda}{2 \omega_{O}^{2}}\left(1+\sqrt{1-4 \frac{\omega_{o}^{2}}{\lambda^{2}}}\right)-f_{o}\right) \exp \left(-\frac{\lambda}{2}\left(1-\sqrt{1-4 \frac{\omega_{o}^{2}}{\lambda^{2}}}\right) t\right)+\left(f_{o}+\frac{k \lambda}{2 \omega_{o}^{2}}\left(1-\sqrt{1-4 \frac{\omega_{o}^{2}}{\lambda^{2}}}\right)\right) \exp \left(-\frac{\lambda}{2}\left(1+\sqrt{\left.1-4 \frac{\omega_{o}^{2}}{\lambda^{2}}\right) t}\right]\right.\right.}
$$

Equation (18) represents mathematically the time dependent strain in the viscoelastic material under study. It predicts the strain versus time relationship of the material studied as a hyper-exponential type function that approaches a maximum value with increasing time.

\section{Numerical Results and Discussion}

Numerical results are carried out in this part for investigating the predictive quality of the model to represent the 
time dependent response of the material studied and the effects of rheological parameters action on the material response. These effects are studied by varying one coefficient while keeping the other constant. The numerical illustrations are investigated at the fixed value of coefficient $K=1$. Numerical results obtained showed the predictive quality of the model. The present model is a four-parameter curve model derived from the Bauer's theory (1984). This theory (Bauer 1984) is based on the determination of the appropriate restoring elastic spring force function $\varphi(\varepsilon)$ that should behave linearly when the deformation $\varepsilon$ becomes small. The nonlinear function $\varphi(\varepsilon)$ chosen here is well suited for the use of the Bauer's approach (1984). As governing evolution equation of the strain, a Lambert-type nonlinear differential equation is obtained. This equation is solved in closed-form solution under the suitable boundary and initial conditions. The model appeared applicable to predict successfully the time dependent deformation response of a variety of viscoelastic materials under a constant loading. A complete characterization of the viscoelastic material studied is taken into account, that is to say, elastic, viscous and inertial nonlinearities are mathematically included in the proposed model. Nevertheless, more experimental results and practical tests are necessary to further verify the feasibility of the model.

\section{Conclusions}

Mathematical models are required to better understand and predict the time dependent behavior of materials under various loading conditions. In this regard a nonlinear theoretical mechanical four-parameter model is developed. The model allowed, according to the resulting results, describing accurately the nonlinear strain versus time relationship in a variety of viscoelastic materials, as a hyper-exponential function or a hyperlogistic-type function that is powerful to represent mathematically any S-shaped experimental data. It is worth noting that the present model is very sensitive to the magnitude of the loading and of the initial strain rate.

\section{References}

Armentano R. L., Barra J. G., Levenson J., Simon A., \& Pichel R. H. (1995). Arterial wall mechanics in conscious dogs. Assessment of viscous, inertial, and elastic moduli to characterize aortic wall behaviour. Circ Res., 76, 468-478.

Bauer R. D., Busse R., Schabert A., Summa Y., \& Wetterer E. (1979). Separate determination of the pulsatile elastic and viscous forces developed in the arterial wall in vivo. Pflügers. Arch., 380, 221-226. http://dx.doi.org/10.1007/BF00582900

Bauer R. D. (1984). Rheological approaches of arteries. Biorheology. Suppl. I, 159-167.

Gamero L. G., Armentano R. L., Barra J. G., Simon A., \& Levenson J. (2001). Identification of Arterial Wall Dynamics in Conscious Dogs. Exp. Physiol., 86, 519-528. http://dx.doi.org/10.1113/eph8602172

Haslach Jr H.W. (2005). Nonlinear viscoelastic, thermodynamically consistent, models for biological soft tissue. Biomechan. Model. Mechanobiol, 3, 172-189. http://dx.doi.org/10.1007/s10237-004-0055-6

Monsia M. D. (2011a). A Hyperlogistic-type Model for the Prediction of Time-dependent Nonlinear Behavior of Viscoelastic Materials. Int. J. Mech. Eng., 4, 1-4.

Monsia M. D. (2011b). A Nonlinear Generalized Standard Solid Model for Viscoelastic Materials. Int. J. Mech. Eng., 4, 11-15.

Monsia M. D. (2011c). A Modified Voigt Model for Nonlinear Viscoelastic Materials. Int. J. Mech. Eng,. 4, $17-20$.

Monsia M. D. (2011d). A Simplified Nonlinear Generalized Maxwell Model for Predicting the Time Dependent Behavior of Viscoelastic Materials. World Journal of Mechanics, 1, 158-167. http://dx.doi.org/10.4236/wjm.2011.13021

Monsia M. D. (2011e). Lambert and Hyperlogistic Equations Models for Viscoelastic Materials: Time-dependent Analysis. Int. J. Mech. Eng., 4, 5-10.

Monsia M. D. (2011f). Modeling the Nonlinear Rheological Behavior of Materials with a Hyper-exponential type Function. Mechanical Engineering Research, Vol. 1, No. 1, 103-109. http://dx.doi.org/10.5539/mer.v1n1p103

Rachev A., Stergiopulos N., \& Meister J. J. (1996). Theoretical Study of Dynamics of Arterial Wall Remodeling in Response to Changes in Blood Pressure. J. Biomechanics, 29, 635-642. http://dx.doi.org/10.1016/0021-9290(95)00108-5

Tan S. C. W., Pan W. X., Ma G., Cai N., Leong K. W., \& Liao K. (2008). Viscoelastic Behaviour of Human Mesenchymal Stem Cells. BMC Cell Biolog, 9: 40. http://dx.doi.org/10.1186/1471-2121-9-40 


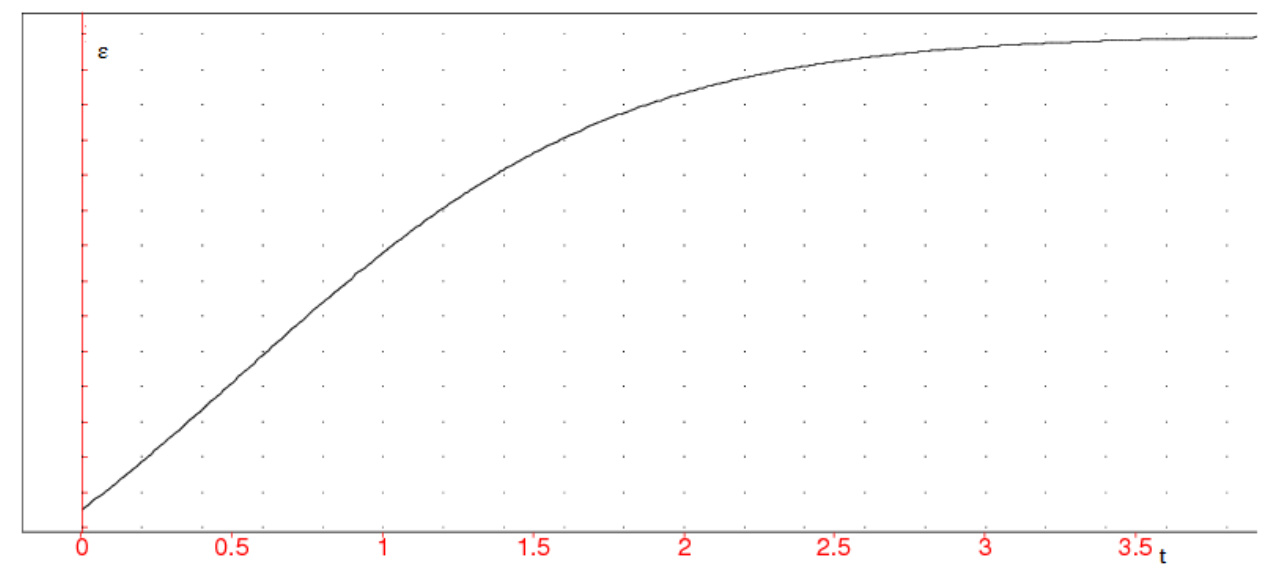

Figure 1. Typical strain versus time curve showing a maximum asymptotical value

Figure 1 exhibits the typical strain versus time curve with an increase until a maximum asymptotical value, obtained from Equation (14) with the fixed value of coefficient at $\lambda=4.32, \omega_{o}=2, f_{o}=1$. Figure 1 shows the capability of the present model to represent analytically and accurately the typical exponential deformation of a variety of viscoelastic materials (Rachev et al. 1996; Haslach Jr 2005; Tan et al. 2008). The time dynamics response of the material is nonlinear, with a nonlinear beginning initial portion. Thus, the plotting shows the S-shaped deformation response of the viscoelastic material investigated.

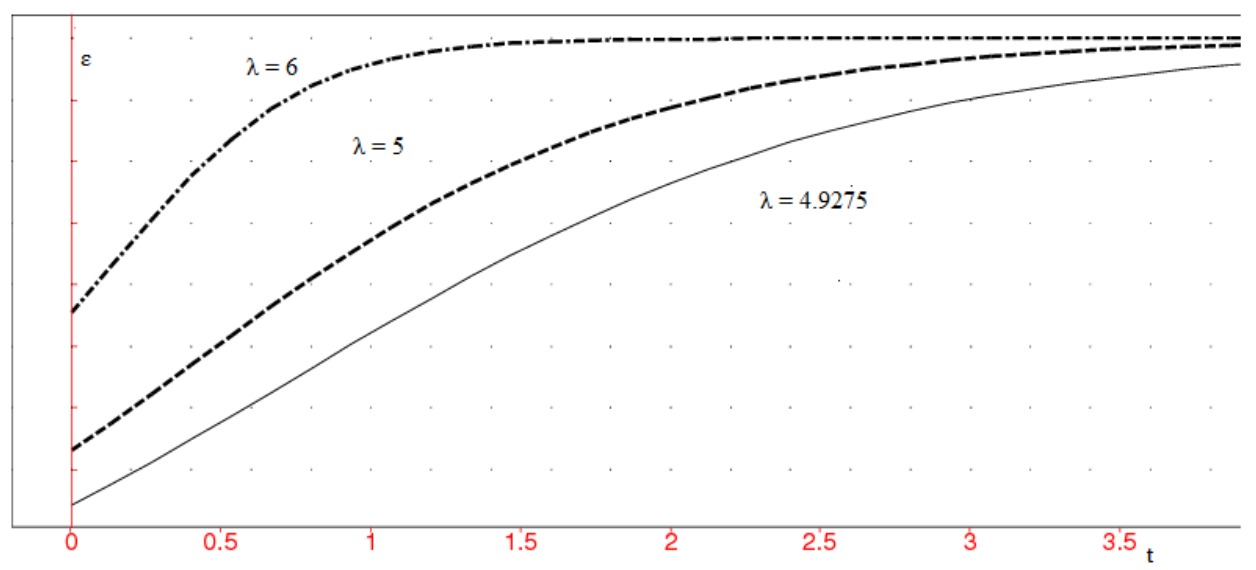

Figure 2. Strain versus time curves illustrating the effects of the damping parameter $\lambda$

Figure 2 shows the effect of the damping coefficient $\lambda$. An increase $\lambda$, reduces the time required to attain the peak strain and increases the initial value of the strain. The solid line corresponds to $\lambda=4.9275$, the dashed line to $\lambda=5$, and the dash-dot line to $\lambda=6$. The other parameters are $\omega_{o}=2.41397, f_{o}=1$. 


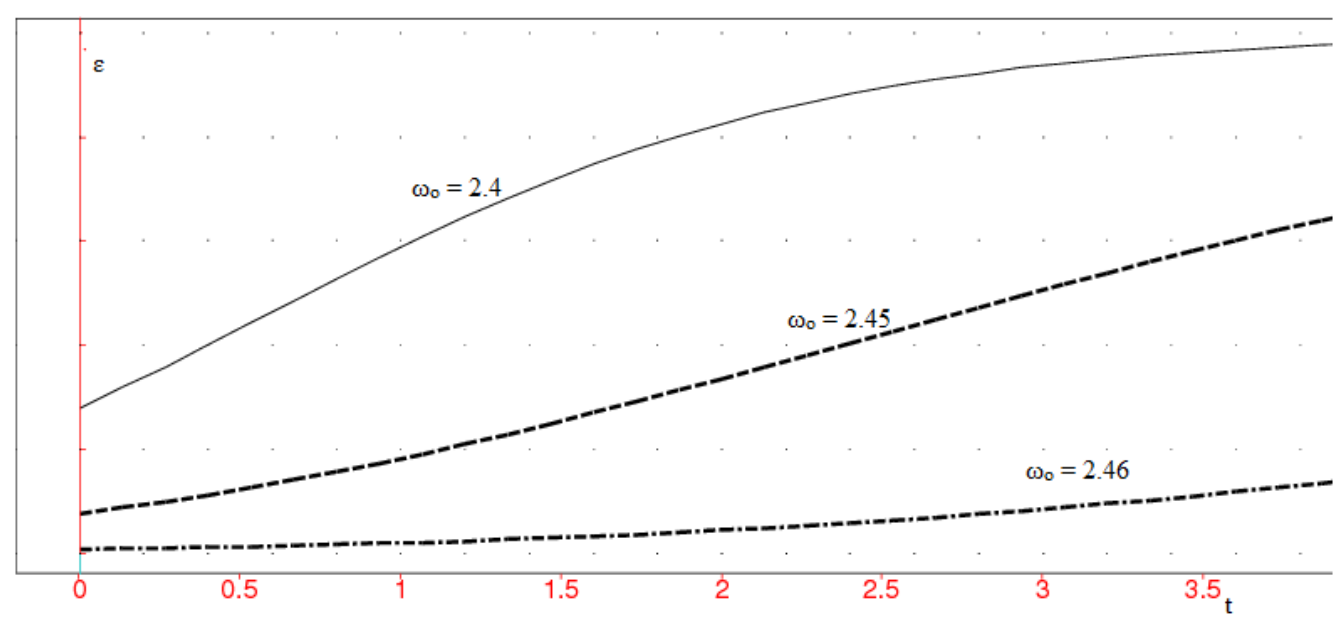

Figure 3. Curves illustrating the effects of the frequency $\omega_{o}$

Figure 3 exhibits the dependence of the strain versus time variation on the frequency coefficient $\omega_{0}$. An increasing $\omega_{o}$, increases the time needed to reach the peak value of the strain and decreases the initial value of the strain. Thus, it is worth mentioning that the effects of the damping coefficient $\lambda$ and the frequency coefficient $\omega_{o}$ affect the strain time curve in opposite directions. The solid line corresponds to $\omega_{o}=2.4$, the dashed line to $\omega_{o}=2.45$, and the dash-dot line to $\omega_{o}=2.46$. The other parameters are $\lambda=4.9275, f_{o}=1$.

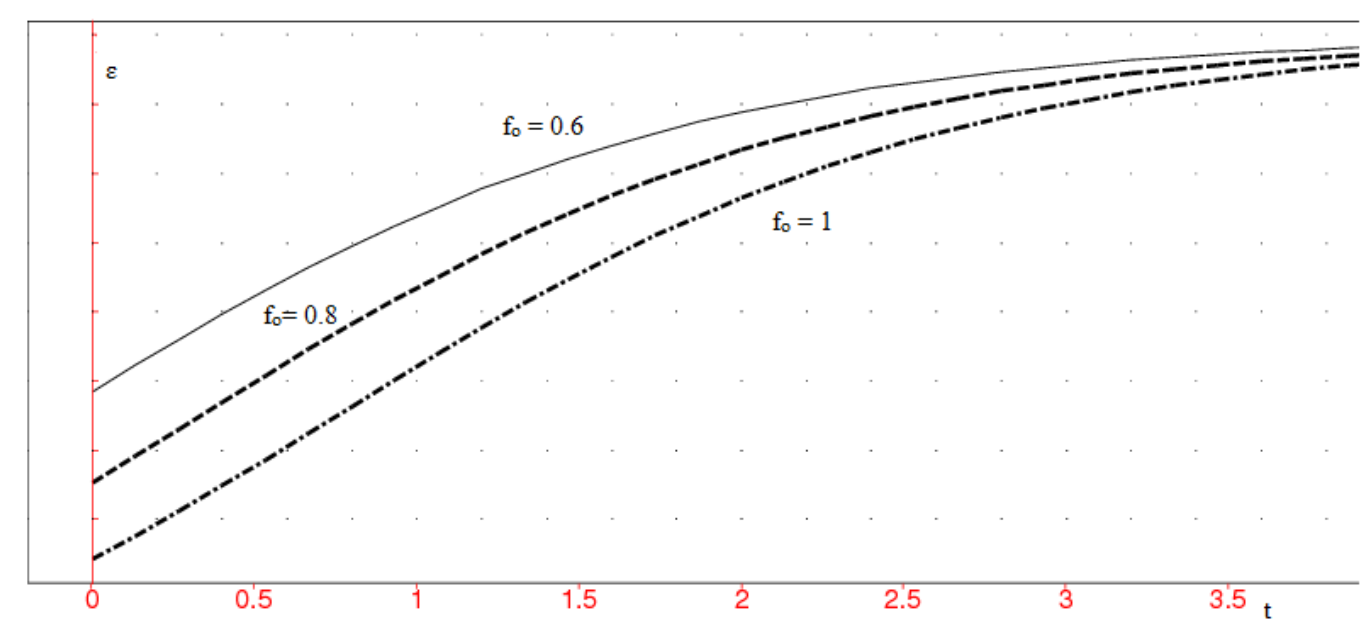

Figure 4. Strain versus time curves at three different values of the coefficient $f_{o}$

We observe from Figure 4 that an increasing $f_{0}$, increases the time needed to attain the peak strain and decreases the initial value of the strain. The solid line corresponds to $f_{0}=0.6$, the dashed line to $f_{0}=0.8$, and the dash-dot line to $f_{0}=1$. The other parameters are $\lambda=4.9275, \omega_{o}=2.41397$. 


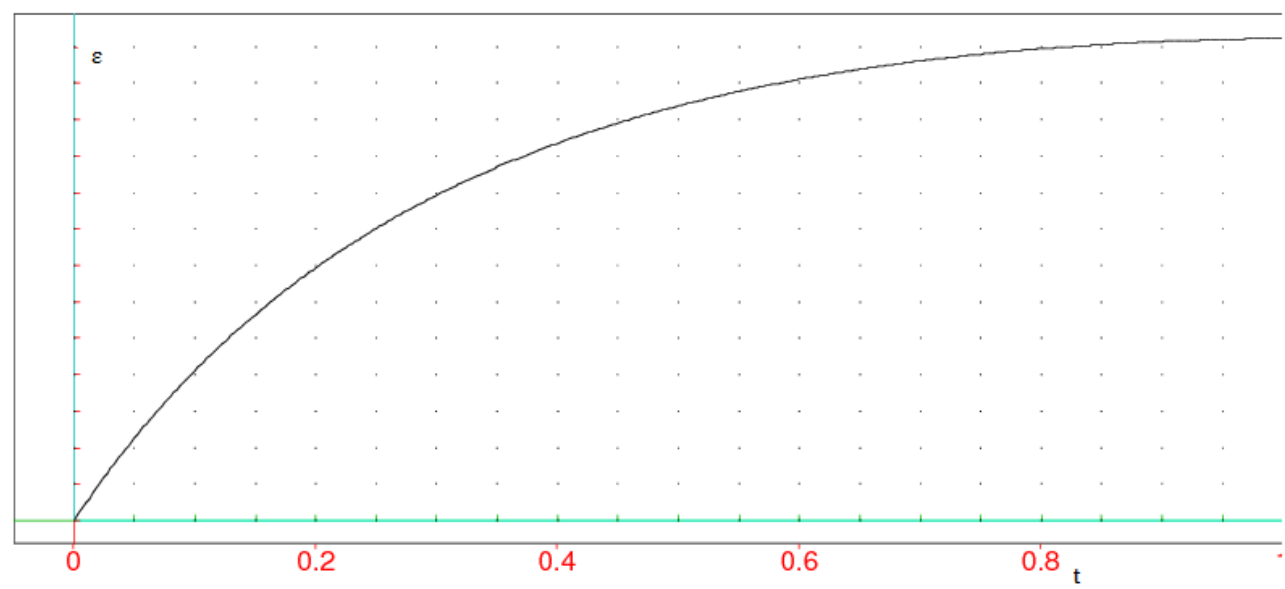

Figure 5. Typical strain-time plotting exhibiting a maximum value

Figure 5 shows the typical time dependent strain curve with an increase until a maximum value, obtained from Equation (18) with the value of coefficients $k=0.2, \lambda=2, \omega_{0}=0.5, f_{0}=1$. It can be noted from Figure 5 the usefulness of the present model to represent mathematically the typical exponential deformation behavior of some viscoelastic materials (Rachev et al. 1996; Haslach Jr 2005; Tan et al. 2008). The model predicts a mechanical response in which the slope declines gradually with increasing time until the failure point at which the slope reduces to zero.

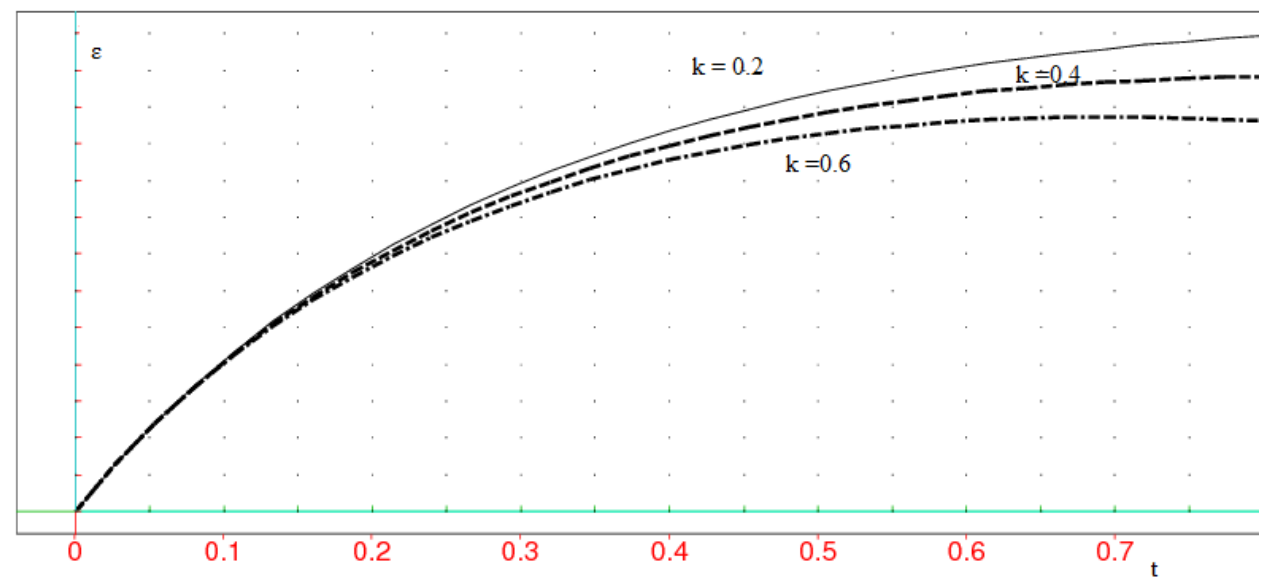

Figure 6. Stress time curves for various values of the coefficient $k$

Figure 6 illustrates the effect of the loading coefficient $k$. An increase $k$, decreases the value of the strain on the time period considered. The slope also decreases with increase $k$. It is worth noting that in the early periods of time, the curves nearly coincide with each other. This fact implies that in this time period the strain versus time variation is approximately stress-independent. This shows then the linear behavior of the model at infinitesimal small strain values. The solid line corresponds to $k=0.2$, the dashed line to $k=0.4$, and the dash-dot line to $k=0.6$. The other parameters are $\lambda=2, \omega_{o}=0.5, f_{0}=1$. 


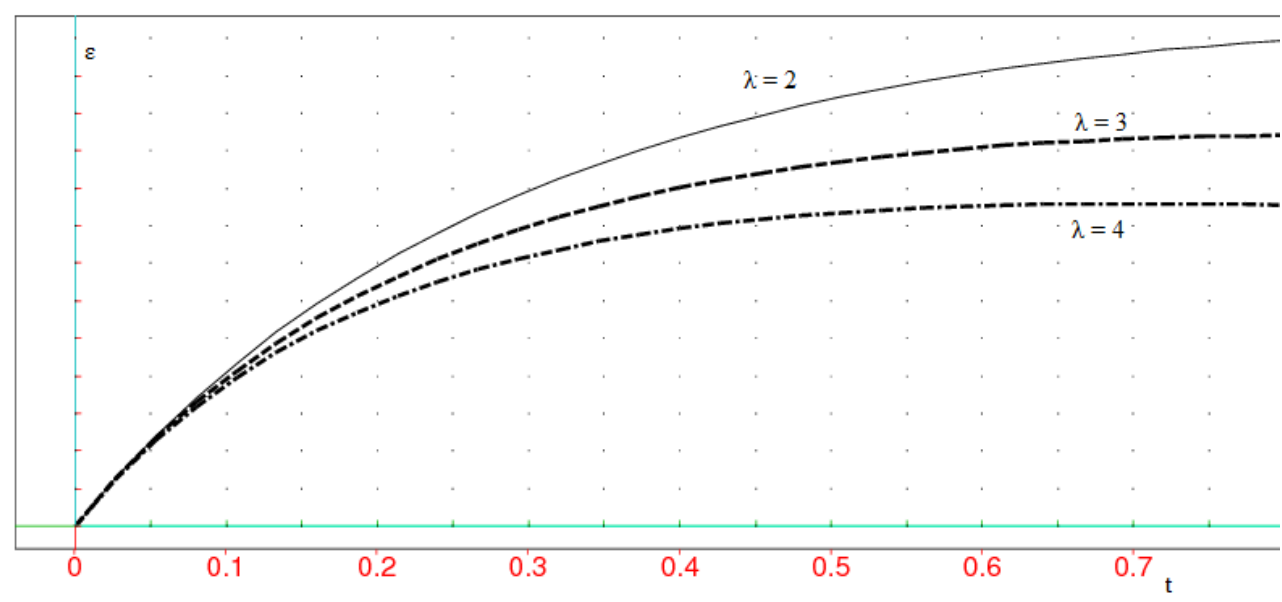

Figure 7. Strain versus time curves at three different values of the damping coefficient $\lambda$

Figure 7 shows the effects of the damping coefficient $\lambda$ change on the strain response. An increase $\lambda$, fast and greatly decreases the value of the strain on the time period considered. The slope also decreases with increasing $\lambda$. The solid line corresponds to $\lambda=2$, the dashed line to $\lambda=3$, and the dash-dot line to $\lambda=4$. The other parameters are $k=0.2, \omega_{o}=0.5, f_{0}=1$.

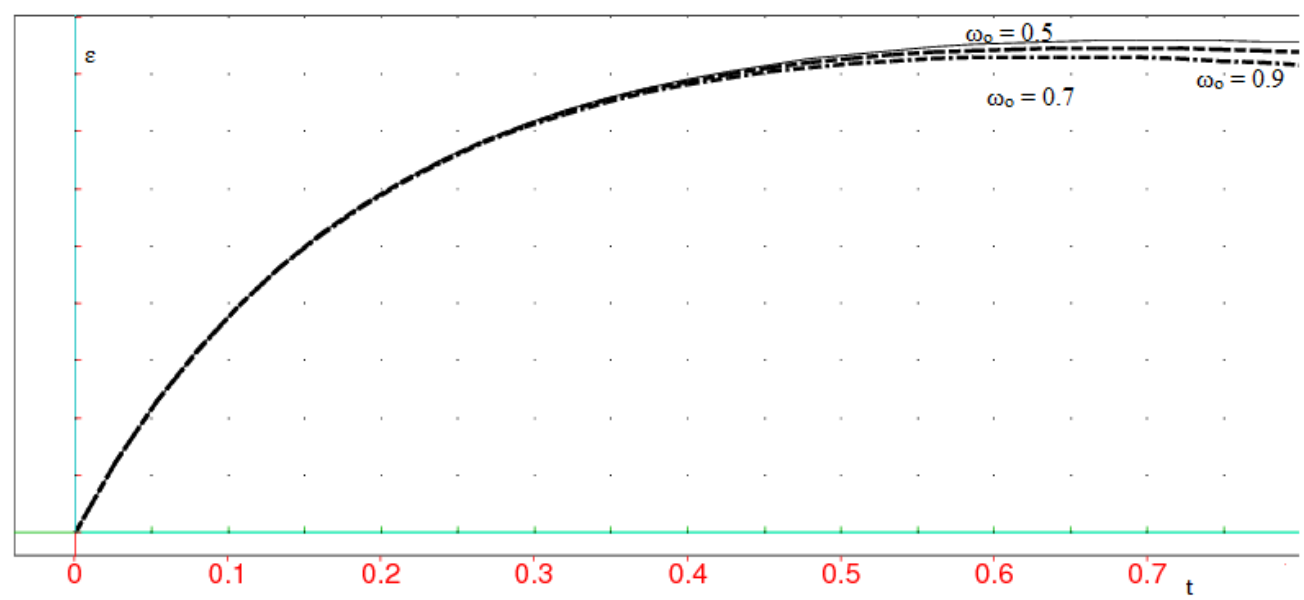

Figure 8. Strain-time curves for three various values of the frequency coefficient $\omega_{0}$

In Figure 8 is shown the dependence of the strain on the frequency coefficient $\omega_{o}$. An increase $\omega_{o}$, slightly decreases the value of the strain on the time period considered. The solid line corresponds to $\omega_{o}=0.5$, the dashed line to $\omega_{o}=0.7$, and the dash-dot line to $\omega_{0}=0.9$. The other parameters are $k=0.2, \lambda=4, f_{0}=1$. 


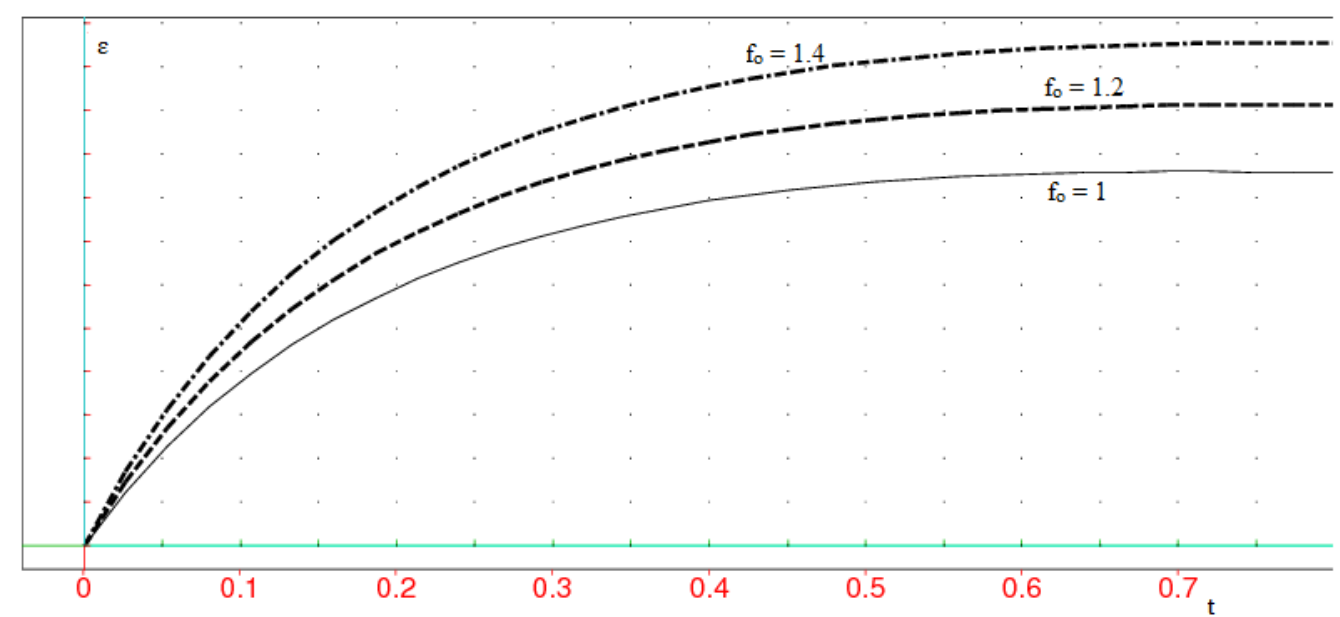

Figure 9. Strain-time curves showing the effect of the initial strain rate $f_{o}$

From Figure 9 we observe that an increasing initial value of the strain rate $f_{o}$, has a significant effect on the strain versus time response. An increasing $f_{o}$, increases significantly and fast the strain value on the time period considered. The slope also increases with increasing $f_{o}$. The solid line corresponds to $f_{o}=1$, the dashed line to $f_{o}=1.2$, and the dash-dot line to $f_{o}=1.4$. The other parameters are $k=0.2, \lambda=4, \omega_{o}=0.5$. 